working population denominator is derived from 2001 Australia Bureau of Statistics census data.

Results There were 4773 initial claims and 416 repeat claims for OCD amongst Victorian workers from January 1985December 2009. The yearly average for initial claims was approximately 9.4 per 100,000 part-time and full-time working Victorians. The mean cost of repeat claims $(\$ 7,556)$ was higher than the mean cost of initial claims $(\$ 4,940)$. These differences between initial and repeat claims are also reflected in the reported days away from work. The mean days away from work for initial claims was 40 and the mean days away from work for repeat claims was 51 .

Conclusions Victorian workers' compensation claims data indicate that the cost and impact of contact dermatitis, as measured by days away from work, increases with repeated workers' compensation claims. Effort needs to be put in place to protect workers from initially developing OCD. For those workers who have developed OCD, a workplace plan needs to be in place for the avoidance or elimination of workplace exposures before the workers return to work.

\section{DIFFERENCES OF MORTALITY RATES BY OCCUPATION IN KOREA: 14 YEARS FOLLOW-UP STUDY}

${ }^{1} \mathrm{He}$ Lee, ${ }^{2} \mathrm{Kim},{ }^{3}$ Kang. ${ }^{1}$ The Catholic University of Korea, Seoul St. Mary's Hospital, Seoul, South Korea; ${ }^{2}$ Occupational Safety and Health Research Institute, Inchoen, South Korea; ${ }^{3}$ Korea Occupational Safety and Health Agency, Seoul Regional Office, Seoul, South Korea

\subsection{6/oemed-2013-101717.124}

Objectives The mortality rates of workers can be influenced by socioeconomic factors as well as occupational ones. This study was performed to observe the mortality rates in various occupations of Korean workers.

Methods We constructed a cohort with workers who have entered the Employment Insurance during 1995-2000. The information of death for the cohort was obtained by matching with database of the National Statistics Office. Nine subcohorts were constructed according to the Korean Standard Occupational Classification. Age-standardised mortality rates (ASR) were calculated.

Results The total cohort included 11,342,816 workers and $141,442,957$ person-years. There were 292,763 deaths during 1995-2000. The ASR for death by all causes was 342.6 per 100,000 in male and 141.6 per 100,000 in female. The highest ASR showed in Agricultural, Forestry and Fishery Workers (M: 563.0, F: 206.0) and followed by Elementary Occupations (M: 499.0, F: 163.4) and Plant, Machine Operators and Assemblers (M: 380.3, F: 157.8). Professionals and related workers showed the lowest ASR (M: 209.1, F: 93.3)Elementary Occupations (M: 146.4, F: 163.4) showed the highest ASR from neoplasm (C00D48). Professionals and related workers (M: 93.2, F: 46.9) and Managers (M: 92.1, F: 41.0) showed the lowest ASR. Agricultural, Forestry and Fishery Workers showed the highest ASR from external causes of death (V01-Y89) (M: 235.1, F: 74.7) and Professionals and related workers showed the lowest ASR (M: 36.2, F: 15.1).

Conclusion We found a profound difference of mortality rate by occupations. Occupations related to low socioeconomic position like Agricultural, Forestry and Fishery Workers and Elementary Occupations showed higher mortality rates and those related to high socioeconomic position such as Managers or Professionals showed lower mortality rates. Further study on mortality rates of workers related to socioeconomic factors as well as workrelated ones is needed.

\section{HEALTH RISKS FROM OCCUPATIONAL EXPOSURE TO EXTREMELY LOW FREQUENCY MAGNETIC FIELDS (ELF- MF) AND ELECTRICAL SHOCKS; AN ANALYSIS IN NOCCA}

${ }^{1} \mathrm{P}$ Slottje, ${ }^{2}$ Kauppinen, ${ }^{3}$ Kromhout, ${ }^{3}$ Huss, ${ }^{4}$ Pukkala, ${ }^{3}$ Vermeulen. ${ }^{1}$ Utrecht University, Utrecht, Nederland; ${ }^{2}$ Finnish Institute of Occupational Health, Helsinki, Finland; ${ }^{3}$ Utrecht University/Institute for Risk Assessment Sciences, Utrecht, Nederland; ${ }^{4}$ Finnish Cancer Registry, Helsinki, Finland

\subsection{6/oemed-2013-101717.125}

Objective Previous epidemiological studies have suggested possible increased health risks of occupational exposure to extremely low frequency magnetic fieds (ELF-MF), in particular cancer (brain, leukaemia), neurological diseases (e.g Amyotrophic Lateral Sclerosis, ALS), and suicide. However, results varied strongly. We aim to assess the association between occupational exposure to ELF-MF and electrical shocks and these priority health outcomes in a large population-based cohort. This could help to increase the knowledge on these health effects and potentially disentangle health risks from ELF-MF and electrical shocks, which has been put forward in particular with respect to ALS.

Methods Case-control risk analyses will be performed in the established Nordic Occupational Cancer (NOCCA) database. The pooled NOCCA population covers over 15 million adult males and females from five Nordic countries with a follow-up of cancer incidence and mortality up to 45 years in 2006. Considering the size and follow-up time of NOCCA, we aim to also study rare outcomes and occupationally exposed females, which will enrich this field of research. Exposure assessment will be based on individual job histories obtained through repeated census data, which will be linked to job exposure matrices for ELFMF and electrical shocks.

Results/Conclusions Results of the risk analyses will be presented at the conference.

\section{THE EPIDEMIC OF CHRONIC KIDNEY DISEASE OF UNCONVENTIONAL ORIGIN IN CENTRAL AMERICA - A CALL FOR TRANSDISCIPLINARY RESEARCH AND ACTION}

${ }^{1} \mathrm{C}$ I Wesseling, ${ }^{2}$ Crowe, ${ }^{3}$ Hogstedt, ${ }^{4}$ Lucas, ${ }^{5}$ Jakobsson, ${ }^{6}$ Wegman. ${ }^{1} / R E T$-Salud, Universidad Nacional (on leave), Heredia, Costa Rica; ${ }^{2}$ IRET, Universidad Nacional, Heredia, Costa Rica; ${ }^{3}$ Karolinska Institute, Stockholm, Sweden; ${ }^{4}$ Department of Public Health and Clinical Medicine, Umeå University, Umeå, Sweden; ${ }^{5}$ Lund university, Lund, Sweden; ${ }^{6}$ University of Massachusetts at Lowell, Lowell, United States of America

\subsection{6/oemed-2013-101717.126}

Background Central Americahas seen a dramatic increase of chronic kidney disease, unexplained by conventional risk factors $(\mathrm{CKDu})$, primarily affecting adult male agricultural labourers. Increases of CKDu are also reported from Sri Lanka and India. Alleged risk factors include environmental toxins. However, observations from Nicaragua and El Salvadorindicate that repeated dehydration due to strenuous work in tropical climate may be a major risk factor that urgently needs to be explored using epidemiologic, experimental and interventional approaches. If heat stress and dehydration prove to be risk factors in themselves, or in combination with others, climate change will dramatically increase the population under risk in the near future. CKD increase in developing countries, regardless 